\title{
Dynamics of solar mesogranulation ${ }^{\star}$
}

\author{
M. Leitzinger ${ }^{1}$, P. N. Brandt ${ }^{2}$, A. Hanslmeier ${ }^{1}$, W. Pötzi ${ }^{1}$, and J. Hirzberger ${ }^{1}$ \\ 1 Institut für Physik, IGAM, Karl-Franzens Universität Graz, Universitätsplatz 5, 8010 Graz, Austria \\ e-mail: martin.leitzinger@aon.at \\ 2 Kiepenheuer-Institut für Sonnenphysik, Schöneckstr. 6, 79104 Freiburg, Germany
}

Received 29 March 2005 / Accepted 3 June 2005

\section{ABSTRACT}

Using a 45.5-h time series of photospheric flow fields generated from a set of high-resolution continuum images (SOHO/MDI) we analyze the dynamics of solar mesogranule features. The series was prepared applying a local correlation tracking algorithm with a $4.8^{\prime \prime} F W H M$ window. By computing 1-h running means in time steps of 10 min we generate 267 averaged divergence maps that are segmented to obtain binary maps. A tracking algorithm determines lifetimes and barycenter coordinates of regions of positive divergence defined as mesogranules (MGs). If we analyze features of lifetimes $\geq 1 \mathrm{~h}$ and of areas $\geq 5 \mathrm{Mm}^{2}$ we find a mean drift velocity of $304 \mathrm{~m} \mathrm{~s}^{-1}$ (with $\pm 1 \sigma$ variation of $180 \mathrm{~m} \mathrm{~s}{ }^{-1}$ ), a mean travel distance of $2.5 \pm 1.8 \mathrm{Mm}$, a mean lifetime of $2.6 \pm 1.8 \mathrm{~h}$, and a $1 / e$ decay time of $1.6 \mathrm{~h}$ for a total of $2022 \mathrm{MGs}$. The advective motion of MGs within supergranules is seen for 50 to $70 \%$ of the long-lived ( $\geq 4 \mathrm{~h}$ ) MGs while the short-lived ones move irregularly. If only the long-lived MGs are further analyzed the drift velocities reduce to $207 \mathrm{~m} \mathrm{~s}^{-1}$ and the travel distances increase to $4.1 \mathrm{Mm}$ on average, which is an appreciable fraction of the supergranular radius. The results are largely independent of the divergence segmentation level.

Key words. convection - Sun: granulation - Sun: photosphere

\section{Introduction}

In 1981, November et al. found a "fairly stationary pattern of cellular flow" from time averages of Doppler velocities measured at disk center. The observed pattern had a spatial scale of 5-10 Mm, a vertical rms velocity amplitude of about $60 \mathrm{~m} \mathrm{~s}^{-1}$ and a lifetime of at least $2 \mathrm{~h}$. They termed this pattern mesogranulation (MG) and speculated that it was the possible "missing" scale between granulation and supergranulation (November et al. 1981).

The question of the origin of MG is still debated. A pattern of so-called active (i.e., repeatedly splitting) granules was found by Oda (1984); its mesh size was comparable to mesogranules. Also Koutchmy \& Lebecq (1986) confirmed the existence of quasi-stationary cells of sizes in the range of 10-15". Dialetis et al. (1988) investigated long-lived granules and found that they are not randomly distributed but that their location formed a meso-scale mesh. A similar pattern with a mean cell size of 7" was seen by Muller et al. (1990); it was formed by large as well as small granules. In an investigation of granular properties on a mesogranular scale Brandt et al. (1991) showed that properties like area, lifetime, brightness and growth rate vary according to where the corresponding granules are located in the MG. Hirzberger et al. (1999) investigated a time series of solar granulation images. They found that small granules (also

^ Full set of maps and movies are only available in electronic form at http://www.edpsciences.org granules of short lifetimes) show a slight tendency to be located in downflow regions and larger granules in upflow regions of the MG. A connection of active granules and the mesogranular flow was also suggested by Müller et al. (2001). They compared the mean distance of long-lived active granules and the mesogranular scale. Also Pötzi et al. (2003) found that granules are not distributed at random, instead "fragmenting, ring-like, and merging" granules are preferentially located in upflow regions (divergence) and "fading" granules in downflow (convergence) regions of the $\mathrm{MG}$.

The question: "Is MG a scale of convection that is distinct from granulation and supergranulation?", is treated by many authors. Using power, phase, and coherence spectra derived from a time series of high spatial resolution spectra of photospheric Fraunhofer lines, Deubner (1989) studied the physical properties of the MG (see Table 1). He reports a "genuine convective flow" in his results. Wang (1989) used long sequences of Dopplergrams and found a typical scale of $7 \mathrm{Mm}$, but no sign of the cellular convection. In an analysis of power spectra, derived from a series of white light images and monochromatic filter scans in two Fraunhofer lines, Straus \& Bonaccini (1997) did not find a "separate regime of mesogranulation distinct from granulation". Ginet \& Simon (1992), Straus et al. (1992) and Hathaway et al. (2000) also find no evidence for a separate mesogranular regime from various analyzing methods, whereas Chou et al. (1991), Bachmann et al. (1997), Ueno \& Kitai (1998) and Domínguez Cerdeña (2003) came to the 
conclusion that there is a convective regime at scales just below supergranulation.

Rieutord et al. (2000) found that "mesogranulation is not a true scale of solar convection but the combination of the effects of both highly energetic granules, which give birth to strong positive divergences" ... "and averaging effects of data processing”. From numerical experiments, Cattaneo et al. (2001) concluded that mesogranules owe their origin to the interaction between granules, while Steiner (2003), also from numerical simulations, found recurrently fragmenting granules that drive a horizontal flow field of mesogranular scale. Similarly, Rast (2003) stated that a simple $n$-body simulation of the interaction of granular downflow plumes was successful at reproducing the observed spatial and temporal scales of both MG and supergranules. Roudier et al. (2003) confirm the suggestions of Straus \& Bonaccini (1997) and Rieutord et al. (2000), that MG is not a "specific scale of convection" ... "but just the largescale extension of granulation". From a two-dimensional analysis of the granular intensity field they found that a "significant fraction of granules" form so-called trees of fragmenting granules, which are very long-lived, a result that was previously found from a one-dimensional time-slice analysis by Müller et al. (2001).

The advective motion of MGs towards the borders of the supergranulation was first described by Muller et al. (1992). Also DeRosa \& Toomre $(1998,2004)$ found convincing evidence in their results from SOHO/MDI Dopplergrams that MGs are advected by the supergranulation. Recently, Shine et al. (2000, henceforth called SSH) analyzed a 45.5-h series of high-resolution continuum images from SOHO/MDI to investigate supergranule and mesogranule evolution. Three statements in the summary of their paper, which are listed below, were the motivation for the present work:

- "Advection of mesogranules from the centers to the boundaries is clearly seen during the 45.5-h run" ... "best seen in high-speed video sequences and space-time slices using the divergence of surface flows."

- "The lifetime of a mesogranule has as an upper limit the transit time from its place of origin within the supergranule to the boundary. This is $4-6 \mathrm{~h}$, depending on the supergranule size. Many of the mesogranules disappear before they arrive at the boundary, and none survives once it reaches it."

- "The advection flow is typically $500-1000 \mathrm{~m} \mathrm{~s}^{-1}$, as observed both by cork motions and from our space-time diagrams."

The main intention of the present work is to use a feature tracking algorithm to determine the tracks and lifetimes of mesogranules. By visualizing the mesogranular motion in the form of vectors we could determine the topography of the advective motions within the supergranule. The data were from SSH in the form of flow fields derived from a local correlation tracking process (for details see $\mathrm{SSH}$ and below) - but needed some further processing in order to be suited for the feature tracking mentioned above.
Table 1. Values of mesogranule lifetime and drift velocity from different authors.

\begin{tabular}{lcc}
\hline \hline & lifetime & $v_{\text {drift }}\left[\mathrm{m} \mathrm{s}^{-1}\right]$ \\
\hline November et al. (1981) & $2 \mathrm{~h}$ & - \\
Deubner (1989) & $\geq 30 \mathrm{~min}$ & 450 \\
Muller et al. (1992) & $3 \mathrm{~h}$ & $300-400$ \\
Ueno \& Kitai (1998) & $\simeq 40 \mathrm{~min}$ & 446 \\
Roudier et al. (1998) & $30-40 \mathrm{~min}$ & 500 \\
& & $(t \geq 70 \mathrm{~min})$ \\
Ploner et al. (2000) & $1-2 \mathrm{~h}$ & - \\
Shine et al. (2000) & $4-6 \mathrm{~h}$ & $500-1000$ \\
present work & $158 \mathrm{~min}$ & 304 \\
\hline
\end{tabular}

\section{Preparation of divergence maps}

The basis of the present work is a time series of 2588 flow fields with a time separation of one minute. These flow fields result from applying a LCT (Local Correlation Tracking) algorithm to a time series of continuum images, obtained by the SOHO/MDI instrument from 17th to 18th of January 1997 (original image size: $1024 \times 500$ pix, where 1 pix $\left.=0.6^{\prime \prime}\right)$. After projection onto a latitude-longitude grid the finally extracted images, that follow the solar rotation, have a size of $304 \times 480$ MDI pixels. For the LCT algorithm a FWHM of 8 pix was used, while the cell centers were spaced 4 pix apart, thus oversampling by a factor of 2 . This cell spacing yields flow fields of $76 \times 120$ cells with a spatial resolution of $4.8^{\prime \prime}$ or $3.5 \mathrm{Mm}$. Because of several gaps in the data set, which add up to about $2.5 \mathrm{~h}$ (141 missing flow fields), we had to interpolate the missing flow fields. Since most of the gaps were short, i.e., 38 out of 49 consisted of 2 missing flow fields and only one was 11 flow fields long, we used a simple interpolation method: the missing flow fields were replaced by the mean of the 30 flow fields before and the 30 flow fields after the gap. Further we rebinned the original flow fields by a factor of 3 in each direction (new field: $228 \times 360$ pix of $1.6^{\prime \prime}$ or $1.16 \mathrm{Mm}$ size each) to obtain a smoother representation of the flow vectors. Next, we computed the divergence of the flow fields and 1-h running means with a time separation of $10 \mathrm{~min}$ (for an example see Fig. 1). This results in a data set of 267 1-h average divergence maps. Next, we applied an unsharp masking to these maps. The parameters for this masking are the width $s$ of the smoothing window and the weight $r$ of the smoothed image, for which we chose $s=12$ pix and $r=0.9$.

To define mesogranular features we generated binary images from the divergence maps; we call them segmented maps. For the segmentation we used a threshold method, and defined every pixel above the threshold as a mesogranular pixel - in the following we call these contiguous positive divergence areas mesogranules.

We selected four levels of the cumulative histogram of the 267 1-h average divergence images as thresholds, i.e., $80 \%$, $85 \%, 90 \%$ and $95 \%$ of the maximum divergence value (Fig. 2 shows the $80 \%$ and the $90 \%$ level). Our main results refer to the reference level of $90 \%$, while the other three levels were 


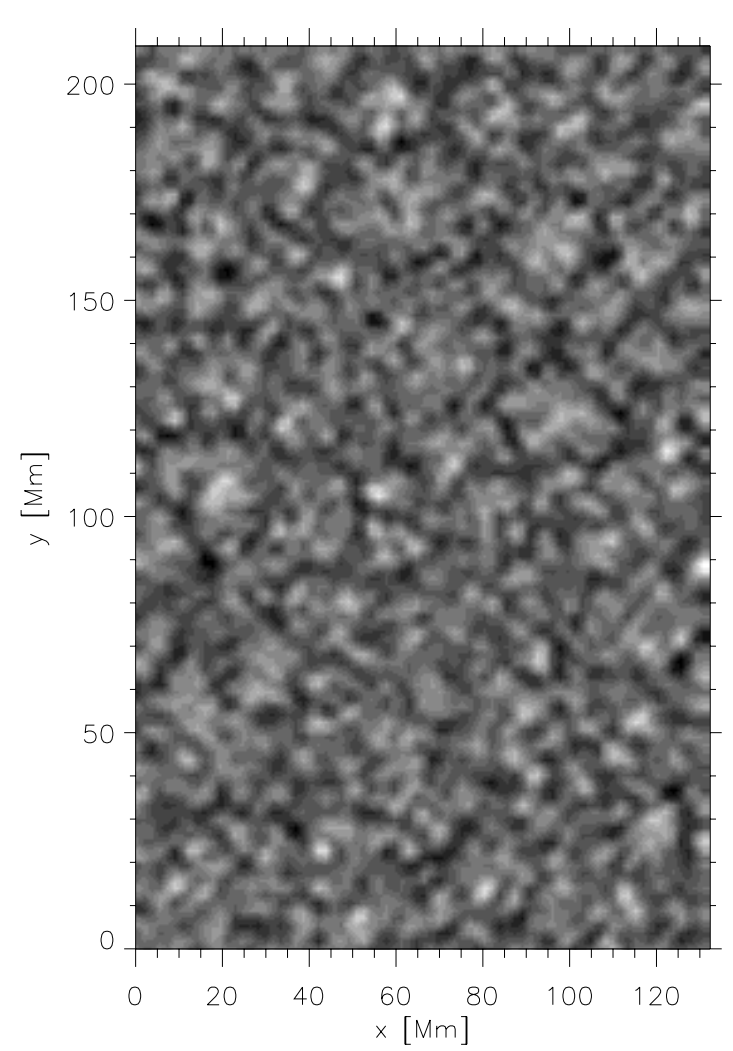

Fig. 1. First one hour average divergence map; bright areas: strong divergence (upflow), dark areas: strong convergence (downflow).

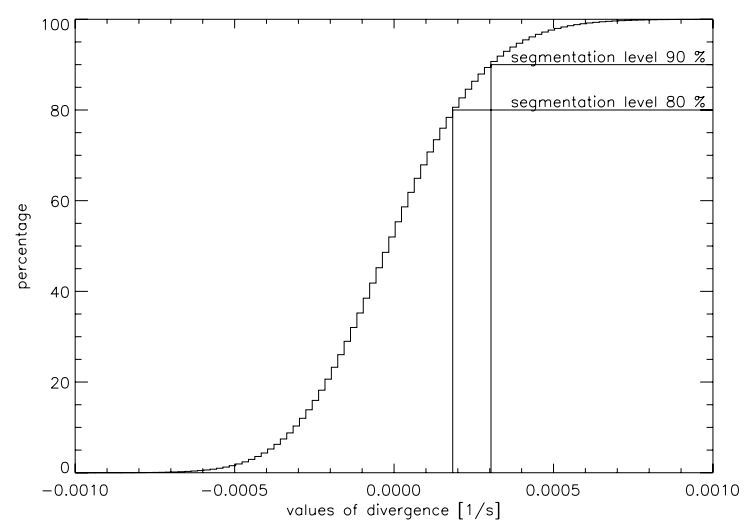

Fig. 2. Mean cumulative histogram of all divergence values of the 267 one-hour averages. The vertical lines show the divergence thresholds at $80 \%$ and $90 \%$. The values of divergence above the threshold are defined as mesogranular pixels.

used to test the stability of the results vs. the variation of this threshold.

Areas of $58 \times 58 \mathrm{Mm}^{2}$ of divergence images and segmented maps are shown in Fig. 3 to demonstrate the influence of the segmentation level on the segmented features. The areas show two main differences between higher and lower segmentation levels: with increasing level both the size and the number of the segmented features decreases. The last step in the preparation process was the identification of the features in order to track them automatically. In each map each feature was assigned its own identification number.

\section{Feature tracking}

The feature tracking algorithm used in this paper is based upon the algorithm of Hirzberger et al. (1999) which was originally developed for the tracking of granules. The algorithm tracks forward and backward in time from a given starting binary map. We chose every fifth segmented map (i.e., time gaps of $50 \mathrm{~min}$ ) as starting point, in order to consider also short-lived features. The adaptation of the algorithm to its present task mainly concerned the interrupt conditions, that select correct followers or predecessors according to the area and the barycenter of the tracked feature; the interrupt conditions are:

- if the new area is larger than twice the previously calculated one, the algorithm stops tracking;

- if the new area is smaller than half the previously calculated one, the algorithm stops tracking;

- if the new barycenter differs more than 3 pixels (or $1.75 \mathrm{Mm}$ ) from the previously calculated one (in $x$ - or $y$-direction) the algorithm stops tracking.

Additionally, a feature tracking algorithm needs rules that define the life and death of mesogranular features; we adopted in our case:

- if an object arises from the background, we call this birth;

- if an object vanishes into the background, we call this death;

- if object No. 1 merges with object No. 2 and the area of No. 2 is bigger than the one of No. 1, we call this the death of object No. 1;

- if an object fragments in two or more parts, the one with the biggest area is maintained.

The results of the algorithm are given in the form of strings, one string for every tracked feature, which include $x / y$ barycenter coordinates, area, map number, and a running index. From the strings, the lifetime, travel distance, travel velocity and mean area can be computed.

\section{Results}

The following results refer to a segmentation level of $90 \%$. Only features with a lifetime of $\geq 1 \mathrm{~h}$ and those with a mean area $\geq 5 \mathrm{Mm}^{2}$ during their evolution were used for the statistical analysis. The latter limit was chosen because the rather large LCT window size of $3.5 \mathrm{Mm}$ advises against investigation of smaller elements. We will show below (cf. Table 2) that the main results to a large extent are independent of the segmentation level.

\subsection{Advective motion and cork maps}

The vectors that show the motion of mesogranular features are plotted in maps (temporally separated by $5 \mathrm{~h}$ for the fullsized maps and $2.5 \mathrm{~h}$ for the cutouts), where the supergranular boundaries are visualized by corks (Figs. 4 and 5). The vectors represent the barycenter motion between birth and death of the MGs. A full set of such maps as well as movies 

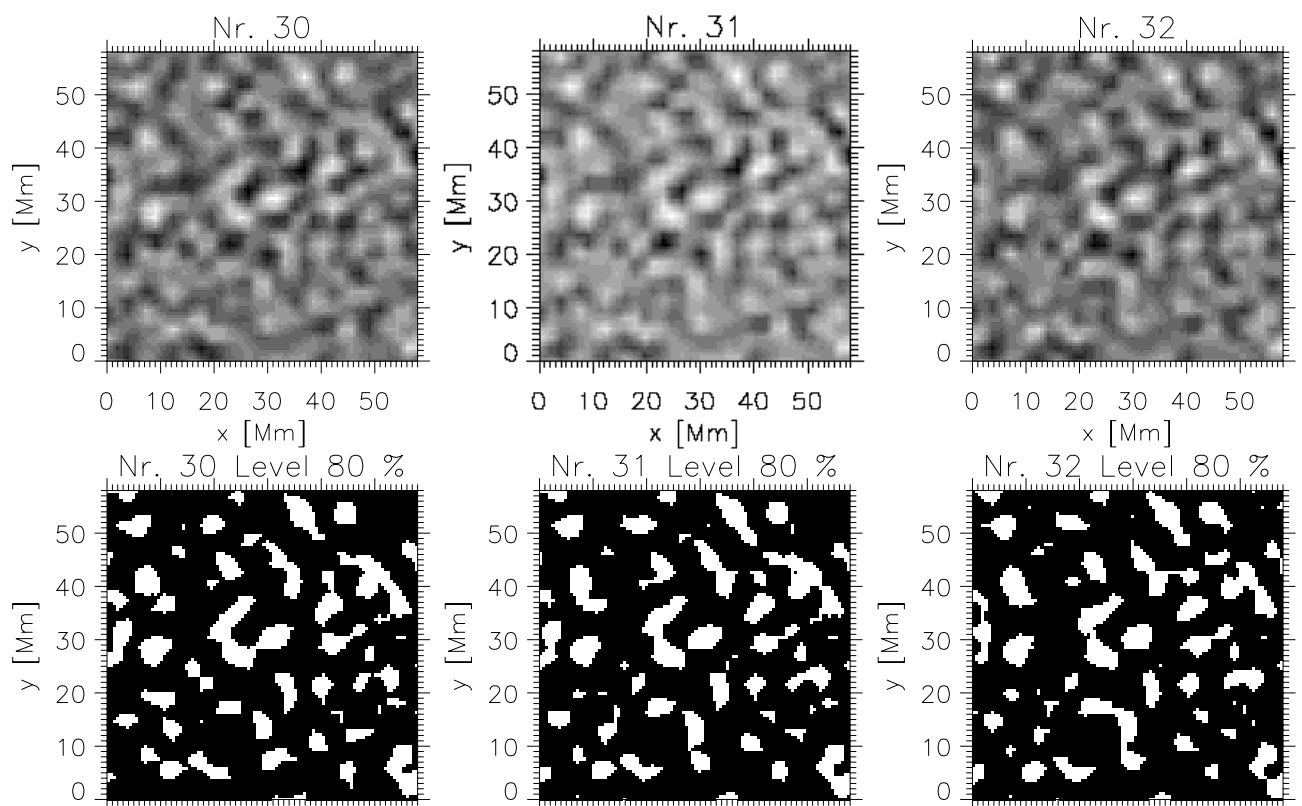

$\begin{array}{llllll}0 & 10 & 20 & 30 & 40 & 50\end{array}$ $\times[\mathrm{Mm}]$
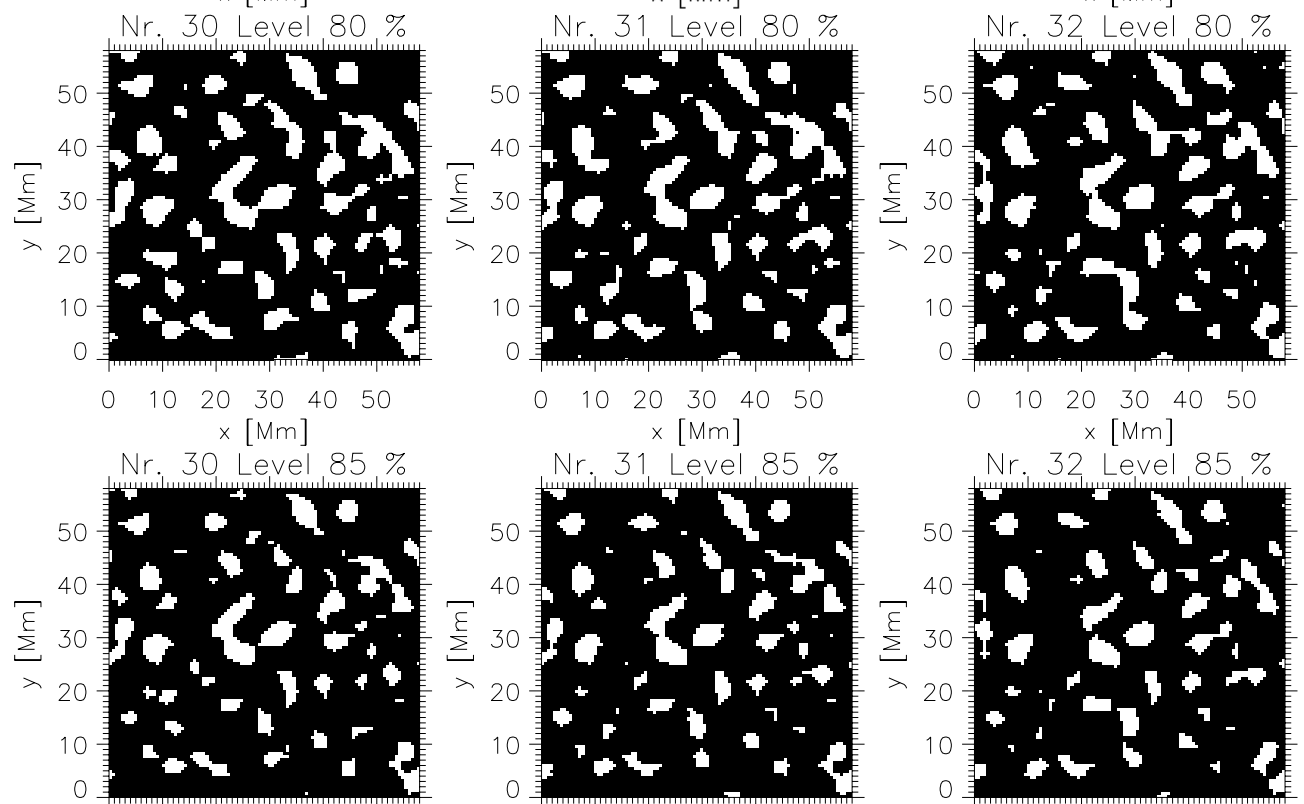

$\begin{array}{llllll}0 & 10 & 20 & 30 \quad 40 \quad 50\end{array}$ $\times[\mathrm{Mm}]$

$0 \quad 1020 \quad 30 \quad 40 \quad 50$ $\times[\mathrm{Mm}]$
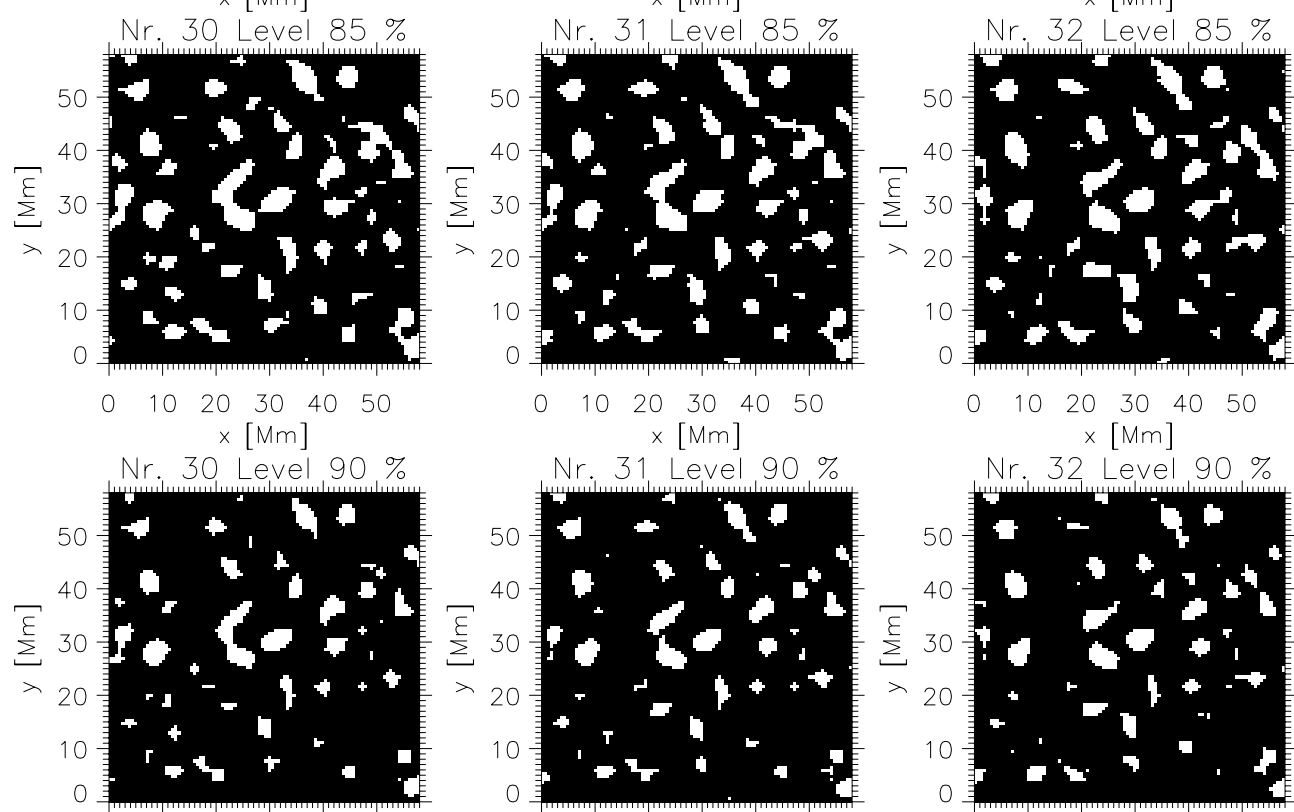

$0 \quad 10 \quad 20 \quad 30 \quad 40 \quad 50$

$\times[\mathrm{Mm}]$

$\begin{array}{llllll}0 & 10 & 20 & 30 & 40 & 50\end{array}$

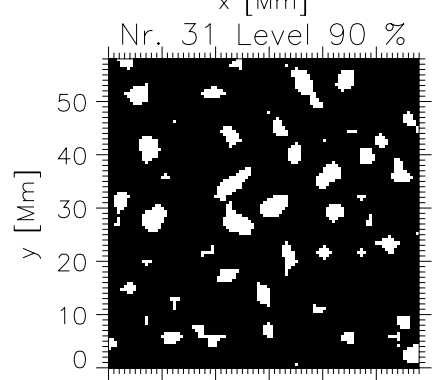

$\times[\mathrm{Mm}]$

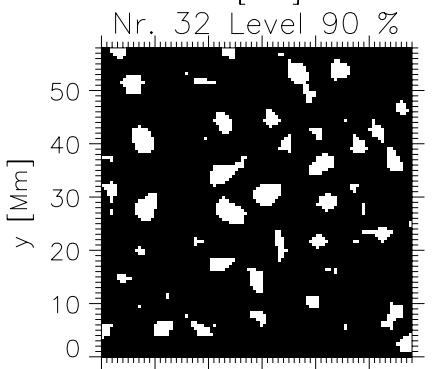

$\begin{array}{llllll}0 & 10 & 20 & 30 & 40 & 50\end{array}$

$\times[\mathrm{Mm}]$

$0 \quad 1020 \quad 30 \quad 40 \quad 50$

$\times[\mathrm{Mm}]$
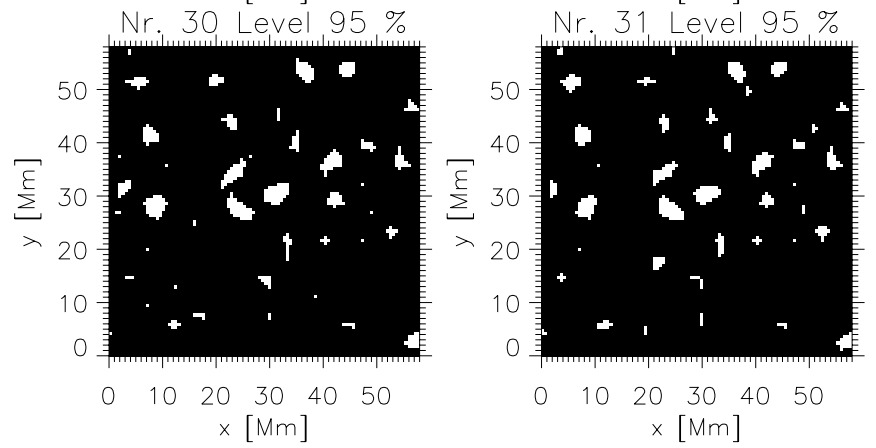

$\begin{array}{llllll}0 & 10 & 20 & 30 & 40 & 50\end{array}$

$\times[\mathrm{Mm}]$

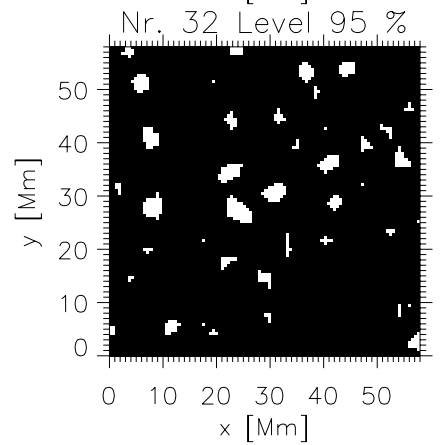

Fig. 3. Areas of $58 \times 58 \mathrm{Mm}^{2}$ of the original divergence (bright: positive) and segmented maps with a cadence of $10 \mathrm{~min}$. The first row shows three successive cutouts of divergence maps. The second to fifth rows show the corresponding segmented maps for segmentation levels $80 \%$ to $95 \%$. The influence of the segmentation level on the segmented features, i.e., decreasing area and number of features with increasing level, is clearly seen. 
Table 2. Mean values of the mesogranular characteristics as function of four segmentation levels; most values are given together with their $\pm 1 \sigma$ scatter around the mean.

\begin{tabular}{lcccc}
\hline \hline segmentation level & $80 \%$ & $85 \%$ & $90 \%$ & $95 \%$ \\
\hline Number of & 2812 & 2483 & 2022 & 1220 \\
tracked mesogranules & & & & \\
Area [Mm $\left.{ }^{2}\right]$ & 16.4 & 12.7 & 9.9 & 7.7 \\
& \pm 9.2 & \pm 6.3 & \pm 4.0 & \pm 2.2 \\
Drift velocity [m/s] & 321 & 314 & 304 & 286 \\
& \pm 193 & \pm 189 & \pm 180 & \pm 172 \\
Lifetime [min] & 131 & 150 & 158 & 151 \\
& \pm 84 & \pm 104 & \pm 107 & \pm 96 \\
Decay time [min] & 65 & 85 & 96 & 99 \\
Travel distance [Mm] & 2.2 & 2.5 & 2.5 & 2.3 \\
& \pm 1.4 & \pm 1.6 & \pm 1.8 & \pm 1.6 \\
\hline
\end{tabular}

of the mesogranular evolution and motion can be found at: http://www . edpsciences.org

The evolution time of the corks is $5 \mathrm{~h}$, sufficient time to advect them to the supergranular boundaries. On the other hand, as one can see from Fig. 7, the lifetimes of MGs vary from $1 \mathrm{~h}$ to $>10 \mathrm{~h}$. Because of this difference in time, the vectors are overplotted on cork maps corresponding to the middle age of the vectors. In some cases, where the lifetime of mesogranules is very long, it can happen that single long-lived mesogranules (or vectors, respectively) cross the supergranular boundaries possibly because of the supergranular evolution over this long time span. However, except for these very rare cases, the maps in Figs. 4 and 5 show that no mesogranular feature crosses the supergranular boundary. Both the long-lived (marked in red) and the far traveling MGs perform a nearly radial advective motion towards the edges of the supergranules; however, there are some exceptions to this behavior - mainly in cases of small or not well defined supergranules. On the other hand, many short-lived features move rather irregularly within the supergranulation and never reach its boundary.

\subsection{Drift velocities}

The drift velocities were computed from the 2022 strings that resulted from the feature tracking (segmentation level 90\%); this was done by applying a polynomial fit (2nd degree) to the $x$ - and $y$-barycenter coordinates. The starting and ending coordinates of the respective polynomial fits were used for computation. We obtain a mean drift velocity of $304 \mathrm{~m} \mathrm{~s}^{-1}$ with a $\pm 1 \sigma$ scatter of $180 \mathrm{~m} \mathrm{~s}^{-1}$ and a median of $272 \mathrm{~m} \mathrm{~s}^{-1}$. The distribution is shown in Fig. 6. Its peak lies near $250 \mathrm{~m} \mathrm{~s}^{-1}$, about $14 \%$ of the MGs exhibit velocities of $\geq 500 \mathrm{~m} \mathrm{~s}^{-1}$ and only $0.2 \%$ move faster than $1000 \mathrm{~m} \mathrm{~s}^{-1}$.

\subsection{Lifetime and decay time}

Figure 7 shows the histogram of the MG lifetimes. The mean lifetime is $158( \pm 107) \mathrm{min}$ or $2.6 \mathrm{~h}$ with a median of $120 \mathrm{~min}$. Of course, the mean value depends strongly on the lowest accepted bin (we adopted 60-70 min here). The distribution shows a maximum at this lowest bin and a very steep decline towards higher values. About $18 \%$ of the MGs live $4 \mathrm{~h}$ and longer, $5.6 \%$ last $\geq 6 \mathrm{~h}$, and we found $12 \mathrm{MGs}$ that lived longer than $10 \mathrm{~h}$. The decline of the number $N$ of MGs with time $t$ follows an exponential law of the form:

$N(t)=N_{o} \cdot \exp -\left(t / T_{D}\right)$

and Fig. 7 shows the fit (solid line) for the parameters $N_{o}=$ 366.6 and $T_{D}=96 \mathrm{~min}$; we conclude from this that the $1 / e$ decay time of MGs is $1.6 \mathrm{~h}$ for the segmentation level of $90 \%$.

\subsection{Area and travel distance}

The area and travel distance histograms of the MGs are shown in Figs. 8 and 9. The mean area of the tracked MGs is 9.9 $( \pm 4.0) \mathrm{Mm}^{2}$ with a median of $9.0 \mathrm{Mm}^{2}$.

However, these values are of limited interest since they depend strongly on the segmentation level (cf. Table 2) while the other mesogranular characteristics do not. The area histogram shows a steep decline from the smallest features that were analyzed (i.e., $5 \mathrm{Mm}^{2}$ ) to values $>30 \mathrm{Mm}^{2}$. The mean travel distance of the MGs is $2.5 \mathrm{Mm}( \pm 1.8)$ with a median of $2.1 \mathrm{Mm}$. Here most of the MGs obviously travel less than 2 to $3 \mathrm{Mm}$ which is only a small fraction of the supergranular radius while only $8.5 \%$ reach distances of $\geq 5 \mathrm{Mm}$, i.e., values comparable to the supergranular radius.

\subsection{Stability of results}

In order to check the stability of the results vs. the variation of the segmentation level we repeated the analysis for segmentation levels of $80 \%, 85 \%, 90 \%$ and $95 \%$. The resulting mean values of drift velocity, lifetime, area and travel distance of the mesogranular features as a function of the segmentation level are listed in Table 2 .

Table 2 shows convincingly that, while the number of tracked MGs drops by a factor of 2.3 and their areas decrease by a factor of 2.1 with increasing segmentation level, the values of drift velocity, lifetime, decay time (except at the lowest level) and travel distance vary only slightly more than $\pm 10 \%$. Moreover, only the drift velocity exhibits a downward trend whereas both lifetime and travel distance go through a flat maximum with increasing segmentation level. This finding gives us confidence in the results - although, of course, they depend on the specific parameters ( $1 \mathrm{~h}$ averaging time and $4.8^{\prime \prime}$ LCT window) used to derive the horizontal velocities.

\subsection{Correlation between results}

How are the various characteristics of the MG related to each other? To answer this question we have created scatter plots of lifetime vs. drift velocity (Fig. 10), of lifetime vs. travel distance (Fig. 11), of lifetime vs. area (Fig. 12) and of travel distance vs. area (Fig. 13).

The first scatter plot (Fig. 10) reveals a clear tendency of the drift velocity to decrease with increasing lifetime. If we 

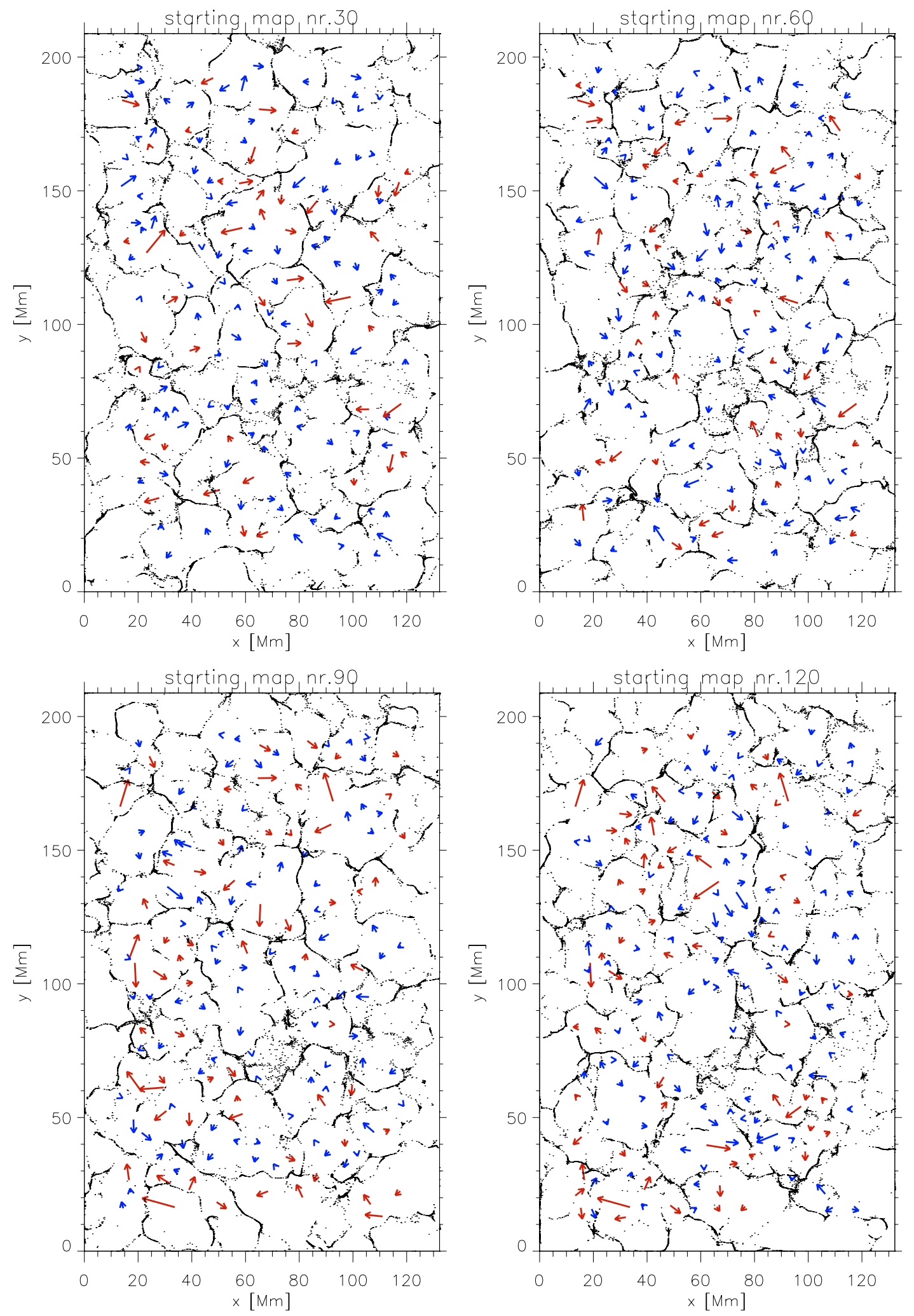

Fig. 4. Four plots of mesogranule trajectories (cadence of $5 \mathrm{~h}$ ) overlayed with cork maps (running time of the corks is $5 \mathrm{~h}$ ). Only mesogranules with a lifetime of $\geq 1 \mathrm{~h}$ were used. Blue-colored vectors correspond to mesogranules with a lifetime $<4 \mathrm{~h}$, whereas red-colored vectors correspond to mesogranules with a lifetime $\geq 4 \mathrm{~h}$.

introduce a limit of $4 \mathrm{~h}$ in order to distinguish between shortlived and long-lived MGs, we find that the short-lived ones drift at velocities between 0.25 and $0.4 \mathrm{~km} \mathrm{~s}^{-1}$ on average, showing a very strong variability between 0 and $\geq 1 \mathrm{~km} \mathrm{~s}^{-1}$, while the long-lived ones drift at about 0.1 to $0.3 \mathrm{~km} \mathrm{~s}^{-1}$ with much smaller variability. Concerning the travel distance, 

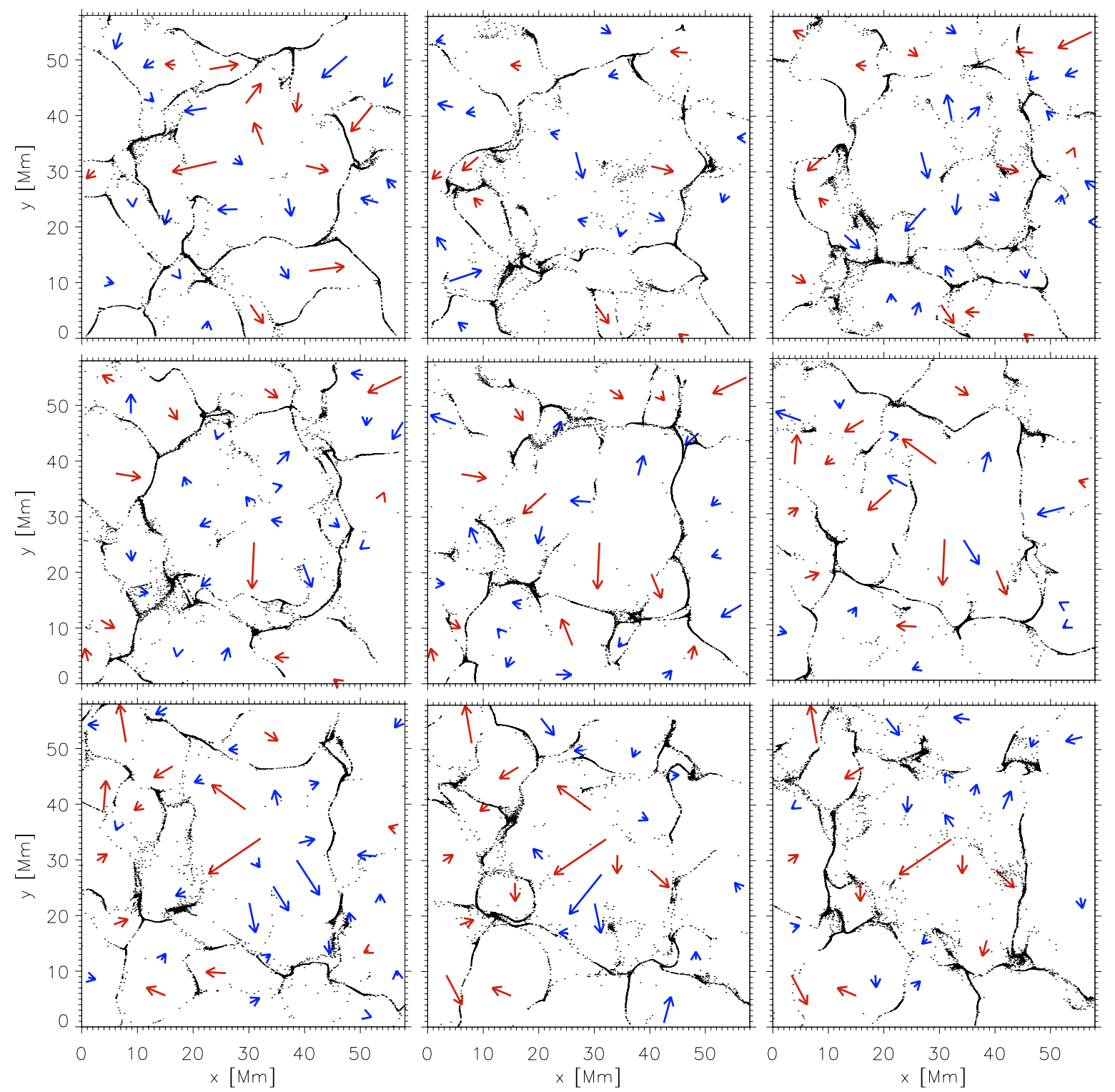

Fig. 5. Cork and vector plots (cadence $2.5 \mathrm{~h}$ ) spanning an area of $58 \times 58 \mathrm{Mm}^{2}$ including one big and several smaller supergranules. The running time of the corks is the same as in Fig. 4 (5 h). Red-colored vectors correspond to mesogranules with a lifetime of $\geq 4 \mathrm{~h}$, the blue-colored vectors to mesogranules with a lifetime $<4 \mathrm{~h}$. The time sequence begins in the upper left corner with a cutout generated from starting image No. 30, proceeding line by line to the lower right corner (starting image No. 150).

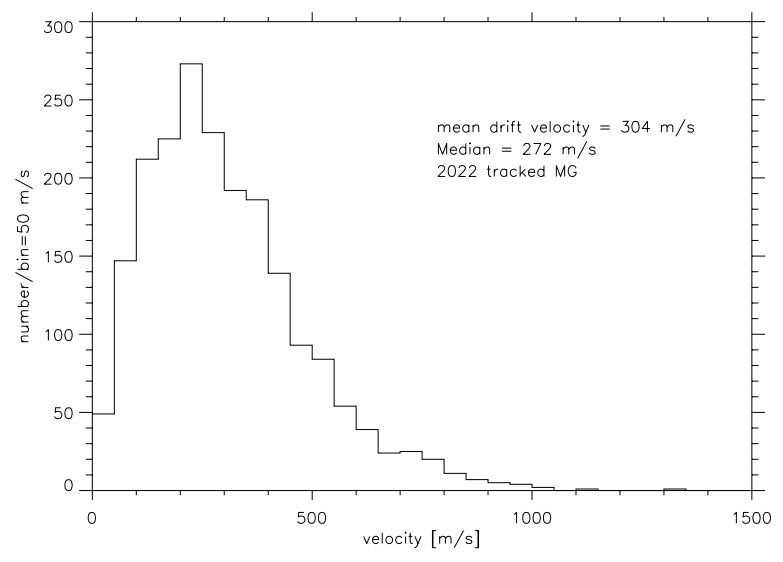

Fig. 6. Histogram of MG drift velocities for the segmentation level of $90 \%$. Only mesogranules of lifetimes $\geq 1 \mathrm{~h}$ and of areas $\geq 5 \mathrm{Mm}^{2}$ were used.
Fig. 11 indicates values of 1 to $3 \mathrm{Mm}$ for the short-lived MGs and a wide range from 3 to more than $10 \mathrm{Mm}$ for the long-lived ones, in other words a clear increase of travel distance with lifetime. The relation between MG area and lifetime (Fig. 12) is characterized by an increase of lifetime with area: the shortlived MGs show areas between 8 and $10 \mathrm{Mm}^{2}$ while the areas of the long-lived ones range from 8 to $>18 \mathrm{Mm}^{2}$. Finally, the relation between MG travel distance and area reveals no specific trend; most of the MGs have small areas $\left(5-10 \mathrm{Mm}^{2}\right)$ and travel 0-3 Mm - a result which could already be read from the histograms (Figs. 8 and 9).

\section{Discussion}

In the following we discuss our results with reference to those quoted by $\mathrm{SSH}$ and their relation to some previous 


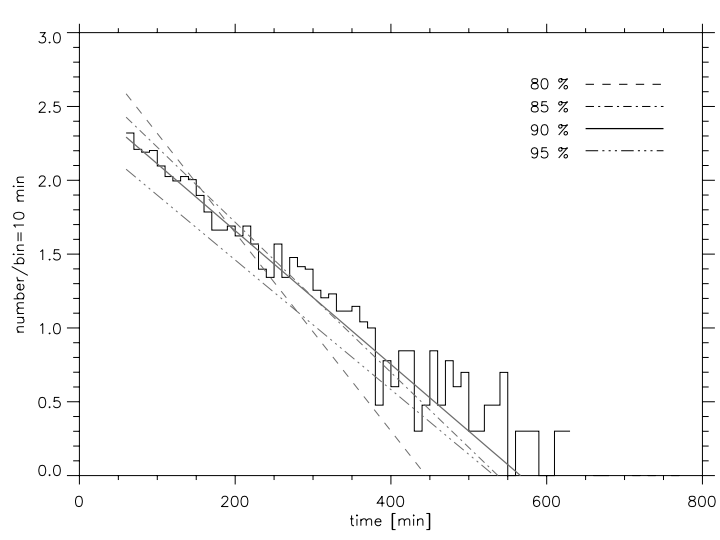

Fig. 7. Number of mesogranules as function of lifetime (in log-lin scale) for the segmentation level of $90 \%$. Only mesogranules of lifetimes $\geq 1 \mathrm{~h}$ and of areas $\geq 5 \mathrm{Mm}^{2}$ were used. The exponential fits for the four segmentation levels are overplotted in different linestyles.

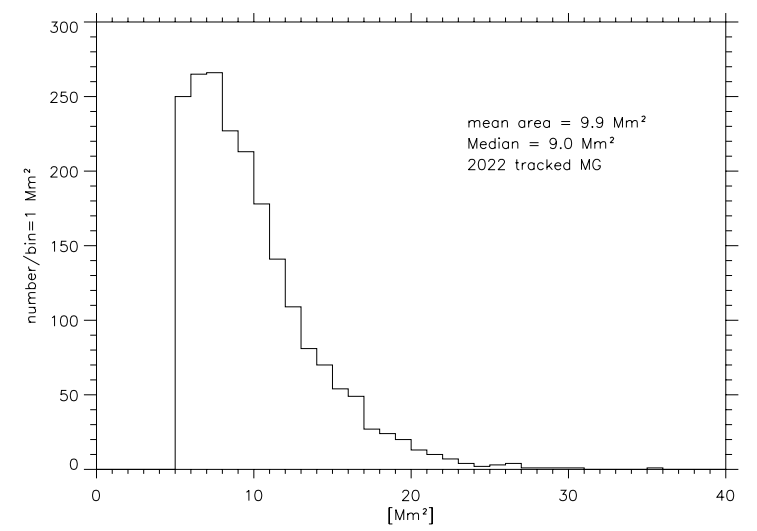

Fig. 8. Histogram of MG areas for the segmentation level of $90 \%$. Only mesogranules of lifetimes $\geq 1 \mathrm{~h}$ and of areas $\geq 5 \mathrm{Mm}^{2}$ were used.

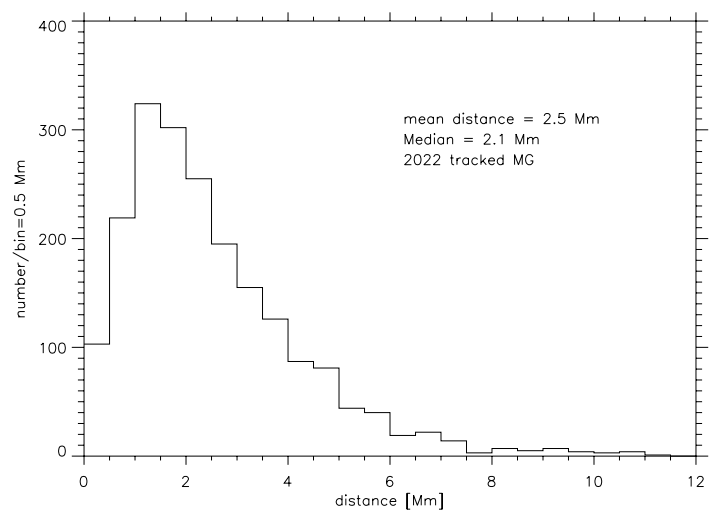

Fig. 9. Histogram of MG travel distances for the segmentation level of $90 \%$. Only mesogranules of lifetimes $\geq 1 \mathrm{~h}$ and of areas $\geq 5 \mathrm{Mm}^{2}$ were used.

measurements of MG characteristics. When comparing these results, each of the quoted results depends in a characteristic way on the specific parameters chosen for the analysis, especially on the spatial and temporal filter that was applied to separate granular from mesogranular properties - the main reason being that the velocity power spectrum shows a continuous

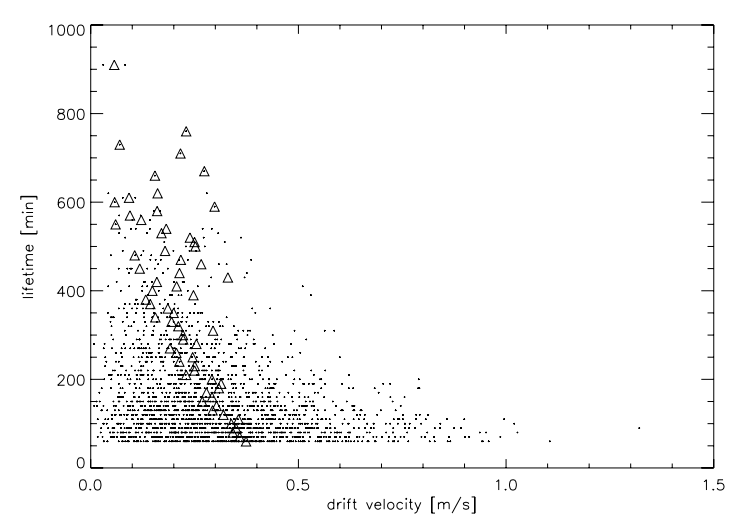

Fig. 10. Scatter plot of MG lifetime vs. drift velocity for the segmentation level of $90 \%$. Triangle symbols mark mean values of velocity for the lifetime bins.

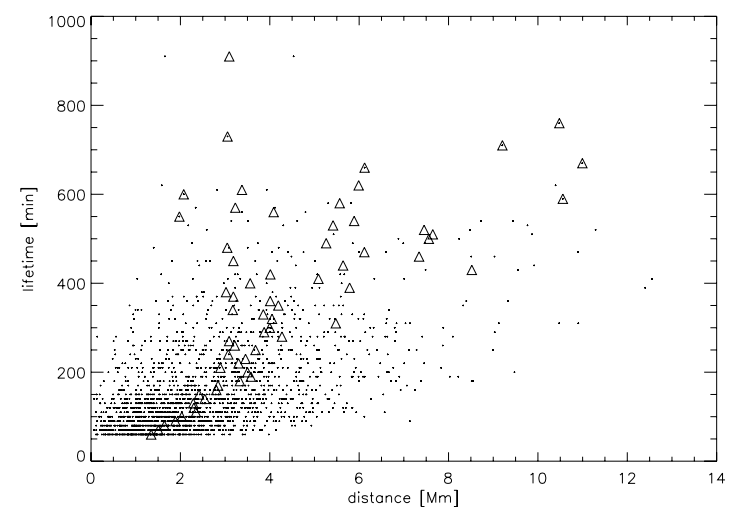

Fig. 11. Scatter plot of MG lifetime vs. travel distance for the segmentation level of $90 \%$. Triangle symbols mark mean values of travel distance for the lifetime bins.

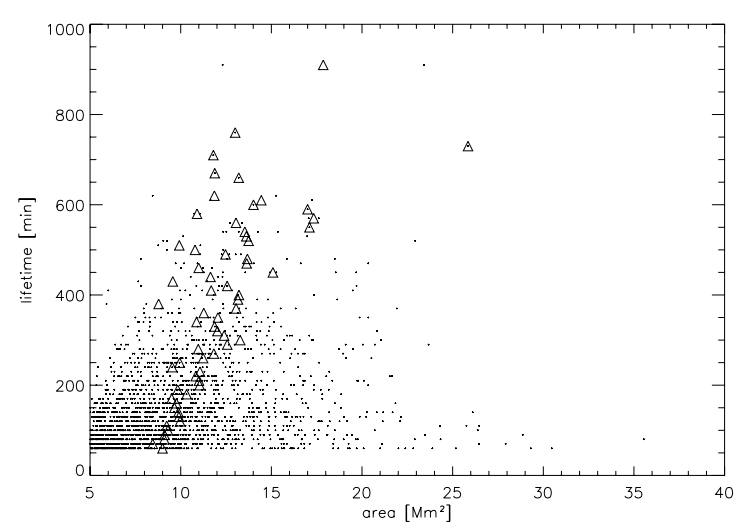

Fig. 12. Scatter plot of MG lifetime vs. area for the segmentation level of $90 \%$. Triangle symbols mark mean values of area for the lifetime bins.

decrease from supergranular down to granular spatial scales, as Hathaway et al. (2000) have demonstrated.

\subsection{Advective motion}

The results presented here in the form of the vector plots, the histograms and the scatter diagrams of the different MG characteristics are only in partial agreement with the results found by SSH. From an inspection of the full set of plots comprising 


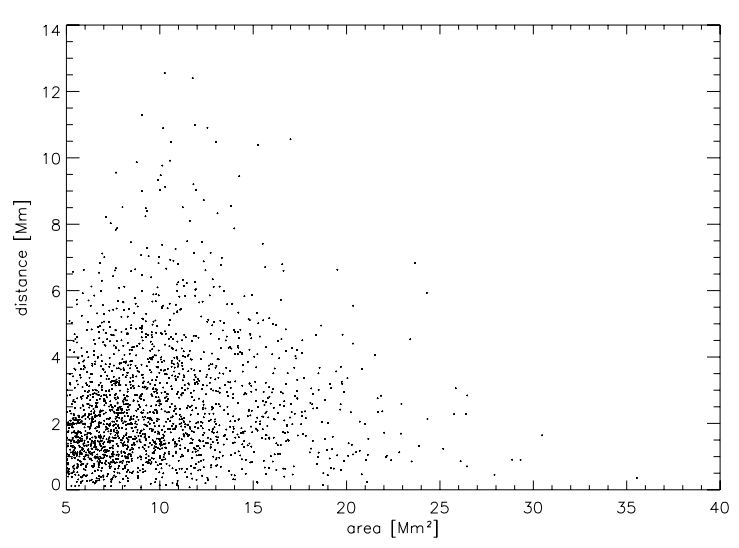

Fig. 13. Scatter plot of MG travel distance vs. area for the segmentation level of $90 \%$.

more than 2000 MGs roughly $50 \%$ of the long-lived ( $\geq 4 \mathrm{~h}$ ) MGs of our analysis indeed show a clear radial motion towards the supergranular boundaries; about $40 \%$ do not show a clear behavior, i.e., move at some angle with respect to the radial direction, while about $10 \%$ fully contradict this pattern. If one distributes one half of the unclear cases (i.e., 20\%) to each of the other two groups about $70 \%$ (i.e., $50 \%+20 \%$ ) of the longlived MGs more or less follow the advective pattern whereas the rest of them do not. On the other hand, only few of the short-lived $(<4 \mathrm{~h})$ ones follow the advective pattern while most of them exhibit rather irregular motions within the supergranulation. With this finding we cannot fully confirm the first statement in the summary of SSH (as cited in Sect. 1). We do agree, however, with SSH with respect to the topology of the advective motions: "many of the MGs disappear before they arrive at the boundary, and none (with the exceptions as discussed in Sect. 4) survives once it reaches it".

\subsection{Lifetime}

Our analysis results in MG lifetimes ranging from the chosen lower limit of $1 \mathrm{~h}$ to values beyond $10 \mathrm{~h}$ with a strong preponderance of short-lived MGs, yielding a mean value of $2.6 \mathrm{~h}$. The large $\pm 1 \sigma$ scatter of $107 \mathrm{~min}$, however, indicates that only the tail of our lifetime distribution overlaps with the high values of 4-6 h quoted by SSH. Table 1 shows that most previous determinations came up with shorter lifetimes than those of SSH, ranging from 30-40 min (Deubner 1989; Ueno \& Kitai 1998; Roudier et al. 1998) to $1-2 \mathrm{~h}$ (November et al. 1981) to $3 \mathrm{~h}$ (Muller et al. 1992). However, one should not forget the very different data material, analysis and filtering methods involved in the various measurements. A better measure than the mean lifetime is the $1 / e$ decay time, because it does not depend on the lower limit of the lifetime values that are included in the analysis; the value of $1.6 \mathrm{~h}$ for the decay time derived here is a novel result that was not mentioned in the literature before.

\subsection{Advection flow velocity}

Choosing a lower limit of the lifetime of $1 \mathrm{~h}$ we obtain a mean MG drift velocity of $304 \mathrm{~m} \mathrm{~s}^{-1}$, with a large $\pm 1 \sigma$ value of
Table 3. Mean characteristics of MGs as function of the lower limit of lifetime; each mean value is given together with its $\pm 1 \sigma$ scatter around the mean.

\begin{tabular}{cccccc}
\hline \hline Limit & Lifetime & $\begin{array}{c}\text { Drift } \\
\text { velocity } \\
{\left[\mathrm{m} \mathrm{s}^{-1}\right]}\end{array}$ & $\begin{array}{c}\text { Travel } \\
\text { distance } \\
{[\mathrm{Mm}]}\end{array}$ & Area & Number \\
& {$[\mathrm{Mm}]$} & & \\
\hline$\geq 1 \mathrm{~h}$ & 158 & 304 & 2.5 & 9.9 & 2022 \\
& \pm 107 & \pm 180 & \pm 1.8 & \pm 4.0 & \\
$\geq 2 \mathrm{~h}$ & 222 & 262 & 3.2 & 10.7 & 1106 \\
& \pm 109 & \pm 150 & \pm 1.9 & \pm 3.7 & \\
$\geq 4 \mathrm{~h}$ & 343 & 207 & 4.1 & 11.9 & 366 \\
& \pm 108 & \pm 111 & \pm 2.3 & \pm 3.6 & \\
$\geq 6 \mathrm{~h}$ & 472 & 176 & 4.9 & 12.9 & 113 \\
& \pm 106 & \pm 99 & \pm 2.8 & \pm 3.6 & \\
& 573 & 163 & 5.5 & 13.7 & 44 \\
& \pm 100 & \pm 90 & \pm 3.0 & \pm 3.8 & \\
\hline
\end{tabular}

$180 \mathrm{~m} \mathrm{~s}^{-1}$. Nevertheless, our mean value is a factor of approx. 2 to 3 lower than the values of $500-1000 \mathrm{~m} \mathrm{~s}^{-1}$ quoted by SSH. The question arises: Could we reconcile our results with those given by $\mathrm{SSH}$ by discarding all features with short lifetimes (regarding them as kind of noise)? Table 3 shows what happens if MGs of short lifetimes are discarded step by step: if we choose $4 \mathrm{~h}$ as a lower limit the resulting 366 MGs show an even smaller drift velocity of $207 \mathrm{~m} \mathrm{~s}^{-1}\left( \pm 111 \mathrm{~m} \mathrm{~s}^{-1}\right)$ and a travel distance of 4.1 Mm $( \pm 2.3 \mathrm{Mm})$; for even longer-lived features the trend continues, i.e., the velocities decrease and the traveled distances increase. From this we conclude that from our feature tracking there is no way to obtain the large velocities quoted by SSH: depending on where we set the lower limit of lifetime we obtain velocities that are a factor of $\approx 2$ to even 5 lower than those given by SSH. Most of the values measured earlier (cf. Table 1) range from 300 to $500 \mathrm{~m} \mathrm{~s}^{-1}$, despite the different methods applied.

One would expect that the advective flow velocities of the MGs, which are measured here at the photospheric level, are closely related to the horizontal velocities measured in supergranules by spectroscopic methods; for these Simon (2001) quotes a range from $200-500 \mathrm{~m} \mathrm{~s}^{-1}$, which is compatible with the range of values of $200-300 \mathrm{~m} \mathrm{~s}^{-1}$ (Table 3 ) found here - especially if one considers the wide spread $\left(1 \sigma \approx 100-180 \mathrm{~m} \mathrm{~s}^{-1}\right)$ of the scatter around the mean. Simon (1967) found "... that the granules tend to move toward the network boundaries, apparently drifting in the direction of the supergranule flow pattern". In the present work we confirm the same pattern for the divergence of this granule drift.

\subsection{Travel distances}

Concerning the travel distances, the scatter plots (Figs. 10 and 11) show that short-lived features have higher drift velocities than long-lived ones; but the long-lived ones, despite their smaller velocities, travel over distances of 3 to $>10 \mathrm{Mm}$ that become comparable to the typical radius of supergranules, which is of the order of 6 to $10 \mathrm{Mm}$ as quoted by Hagenaar et al. (1997) and by DeRosa \& Toomre (2004); compared to this the 
supergranule radius values of $10-15 \mathrm{Mm}$ quoted by SSH seem large. The mean values of Table 3 show that in our analysis MGs living $\geq 4 \mathrm{~h}$ travel $4.1 \mathrm{Mm}( \pm 2.3 \mathrm{Mm})$, those living $\geq 8 \mathrm{~h}$ travel 5.5 Mm $( \pm 3.0 \mathrm{Mm})$. On the other hand, if one multiplies SSH's lower values of the flow velocity $\left(500 \mathrm{~m} \mathrm{~s}^{-1}\right)$ and lifetime $(4 \mathrm{~h})$ one obtains a travel distance of $7.2 \mathrm{Mm}$, whereas multiplying their two higher values (i.e., $1 \mathrm{~km} \mathrm{~s}^{-1}$ and $6 \mathrm{~h}$ ) yields $21.6 \mathrm{Mm}$. While the former value seems still acceptable with respect to the supergranular radius mentioned above the latter seems too large, especially in view of the fact that "many of the MGs disappear before they arrive at the boundary ...", a fact that reduces the mean value computed for the ensemble.

\subsection{Cork motion}

In Fig. 5 of their publication SSH show the distribution of the flow velocities derived from their LCT analysis of granule conglomerates: it has a peak around $450 \mathrm{~m} \mathrm{~s}^{-1}$ and shows a broad tail reaching beyond $1000 \mathrm{~m} \mathrm{~s}^{-1}$. In an analysis of a high-resolution granulation series observed at Pic du Midi Roudier et al. (1999) found a velocity histogram that peaks around $0.6 \mathrm{~km} \mathrm{~s}^{-1}$ and reaches up to $2.5 \mathrm{~km} \mathrm{~s}^{-1}$. The difference between the findings of SSH and Roudier et al. may be explained by the large difference in the FWHM of the LCT windows: while Roudier et al. used a window of $0.7^{\prime \prime}$ and thus were able to track single granules, SSH's window size was $4.8^{\prime \prime}$ and was, therefore, only capable of tracking groups of granules.

Tracer particles (corks) traveling at, e.g., $0.5 \mathrm{~km} \mathrm{~s}^{-1}$ for 4-6 h cover the supergranular radius; therefore the statement by SSH is very appropriate: "Corks (tracers) are cleared from the interiors of medium $(20 \mathrm{Mm})$ supergranules in $4 \mathrm{~h}$, and from large ones $(30 \mathrm{Mm})$ in 6 h". Our Figs. 4 and 5, produced with cork travel times of $5 \mathrm{~h}$, confirm this, except in regions where magnetic fields are present, as is the case in Fig. 4 in a broader region around $x=70 \mathrm{Mm}, y=70 \mathrm{Mm}$. However, the motion of the corks represented by the flow fields is not necessarily the same thing as the motion of its derivative, the divergence, which by definition represents the MG. This is consistent with our finding of MG drift velocities which are about a factor of 2 lower than the typical cork motion, as determined from tracking granules or groups of granules.

\section{Conclusion}

From our re-analysis of a 45.5-h set of flow fields that were derived by Shine et al. (2000) by tracking groups of granules on SOHO/MDI continuum images we find that 50 to $70 \%$ of the MGs of lifetimes $\geq 4 \mathrm{~h}$ exhibit a more or less radial motion within supergranules; shorter lived mesogranules travel in random directions. We find a wide range of sizes, lifetimes, travel distances and velocities in our sample of mesogranules. If the analysis is limited to MGs of lifetimes $\geq 1 \mathrm{~h}$ and of areas $\geq 5 \mathrm{Mm}^{2}$ we find the following mean values: lifetime 158 $( \pm 107) \mathrm{min}$, travel distance $2.5( \pm 1.8) \mathrm{Mm}$, and drift velocity $304( \pm 180) \mathrm{m} \mathrm{s}^{-1}$. A novel result is the $1 / e$ decay time of $1.6 \mathrm{~h}$ found here. If the analysis is limited to MGs of lifetimes $\geq 4 \mathrm{~h}$ the resulting travel distances increase to $4.1( \pm 2.3) \mathrm{Mm}$ while the corresponding drift velocities reduce to $207( \pm 111) \mathrm{m} \mathrm{s}^{-1}$.
The travel distances thus are $1 / 3$ to $1 / 2$ of the commonly accepted values of the supergranular radius of 6-10 Mm (cf. Hagenaar et al. 1997; DeRosa \& Toomre 2004) and the drift velocities are comparable to the horizontal flow velocity of the supergranulation as determined spectroscopically (Simon 2001); the latter fact suggests the interpretation that the MG elements are carried along with the horizontal supergranular flow. In their recent careful investigation of supergranule evolution, DeRosa \& Toomre (2004) tracked by LCT the proper motion of meso-scale elements after high-pass filtering MDI Dopplergrams and thus determined the supergranular pattern from the horizontal outflows observed.

The differences between our results and those of SSH mainly may be due to the fact that their visual inspection of movie sequences and space-time diagrams may have preferred other sub-sets of features and thus have been more prone to selection effects than the more objective method applied here. The lifetimes found by other authors (cf. Table 1) range from $30 \mathrm{~min}$ to $3 \mathrm{~h}$ and their drift velocities are in the range $300-500 \mathrm{~m} \mathrm{~s}^{-1}$ which is in good agreement with our values, especially when one keeps in mind that due to the specific method applied here (large LCT window of 4.8", 1-h running means) the slowly evolving large-scale component of the mesogranulation was studied. On the other hand, the large field of view and the long duration of $45.5 \mathrm{~h}$ with a 1-min cadence of the observational data give us strong confidence in the statistical stability and significance of the results.

Acknowledgements. We thank R. A. Shine for supplying the prereduced data and M. Stix as well as O. Steiner for helpful comments on the manuscript.

\section{References}

Bachmann, K. T., Khatri, G., Petitto, J. M., \& Hathaway, D. H. 1997, Amer. Astron. Soc. Meet., 191, 7404B

Brandt, P. N., Ferguson, S., Scharmer, G. B., et al. 1991, A\&A, 241, 219

Cattaneo, F., Lenz, D., \& Weiss, N. 2001, ApJ, 563, L91

Chou, D.-Y., LaBonte, B. J., Braun, D. C., \& Duvall, T. L. 1991, ApJ, 372,314

DeRosa, M. L., \& Toomre, J. 1998, Proceedings of the SOHO 6/GONG 98 Workshop, Boston, USA, 1-4 June 1998, ESA SP-418, 753

DeRosa, M. L., \& Toomre, J. 2004, ApJ, 616, 1242

Deubner, F.-L. 1989, A\&A, 216, 259

Dialetis, D., Macris, C., Muller, R., \& Prokakis, T. 1988, A\&A, 204, 275

Domínguez Cerdeña, I. 2003, A\&A, 412, L65

Ginet, G. P., \& Simon, G. W. 1992, ApJ, 386, 359

Hagenaar, H. J., Schrijver, C. J., \& Title, A. M. 1997, ApJ, 481, 988

Hathaway, D. H., Beck, J. G., Bogart, R. S., et al. 2001, Sol. Phys., 193, 299

Hirzberger, J., Bonet, J. A., Vázquez, M., \& Hanslmeier, A. 1999, ApJ, 515, 441

Koutchmy, S., \& Lebecq, C. 1986, A\&A, 169, 323

Lawrence, J. K., Cadavid, A. C., \& Ruzmaikin, A. 2001, Sol. Phys., 202, 27

Muller, R., Roudier, Th., \& Vigneau, J. 1990, Sol. Phys., 126, 53

Muller, R., Auffret, H., Roudier, Th., et al. 1992, Nature, 356, 322 
Müller, D. A. N., Steiner, O., Schlichenmaier, R., \& Brandt, P. N. Roudier, Th., Lignières, Rieutord, M., Brandt, P. N., \& Malherbe, J. 2001, Sol. Phys., 203, 211 M. 2003, A\&A, 409, 299

November, L. J., Toomre, J., Gebbie, K., \& G., Simon 1981, ApJ, 245, L123

Oda, N. 1984, Sol. Phys., 93, 243

Shine, R. A., Simon, G. W., \& Hurlburt, N. E. 2000, Solar Phys., 193, 313

Simon, G. W. 1967, Z. Ap., 65, 345

Ploner, S. R. O., Solanki, S. K., \& Gadun, A. S. 2000, A\&A, 356, 1050

Simon, G. W. 2001, in Encyclopedia of Astron. and Astrophys., ed. P. Murdin, 3, 2668

Pötzi, W., Brandt, P. N., \& Hanslmeier, A. 2003, Hvar Obs. Bull., 27, 39

Rast, M. P. 2003, ApJ, 597, 1200

Steiner, O. 2003, in Modelling of Stellar Atmospheres, ed. N. E. Piskunov, W. W. Weiss, \& D. F. Gray, IAU Symp., 210, CD-ROM

Rieutord, M., Roudier, Th., Malherbe, J. M., \& Rincon, F. 2000, A\&A, 357, 1063

Straus, Th., Deubner, F.-L., \& Fleck, B. 1992, A\&A, 256, 652

Straus, Th., \& Bonaccini, D. 1997, A\&A, 324, 704

Ueno, S., \& Kitai, R. 1998, PASJ, 50, 125

Roudier, Th., Malherbe, J. M., Vigneau, J., \& Pfeiffer, B. 1998, A\&A, Wang, H. 1989, Sol. Phys., 123, 21 330,1136 